\title{
THE SOLUTION OF HARMONIC EQUATIONS BY MEANS OF DEFINITE INTEGRALS
}

\section{H. BATEMAN}

Darboux devotes a chapter of his famous Théorie des Surfaces to the study of partial differential equations related to some equations of mathematical physics that have sets of simple solutions of type $X(x) Y(y)$. Such equations are called harmonic equations and are of great interest on account of the various ways in which they can be reduced to the harmonic form and solved by definite integrals. The reduction problem has already been more or less solved, but it cannot be said that the definite integral problem has been fully discussed.

It is known, for instance, that with certain special forms of the functions $a(\theta), b(\theta), h(\theta), f(\theta)$ an integral of type

$$
z=\int_{0}^{\pi} F\left(a x^{2}+2 h x y+b y^{2}\right) f(\theta) d \theta
$$

may satisfy the harmonic equation

$$
r+t+u p / x+v q / y=0, \quad u=2 k+1, v=2 m+1,
$$

where $u$ and $v$ are constants and $p, q, r, s, t$ denote the partial derivatives of $z$ of the first two orders. For general values of $u$ and $v$ this equation is of interest in the study of solutions of the wave equation and Laplace's equation in four variables. When $u$ and $v$ are integers, the equation arises in the study of symmetrical solutions of Laplace's equation in $N$ variables, in the study of the stream function of hydrodynamics and of various functions which occur in the theory of elasticity.

To find all the possible forms of $a(\theta), b(\theta), h(\theta), f(\theta)$ when it is assumed that the integral can be differentiated in the usual way, we must discover how to satisfy the equation

$$
\begin{aligned}
0= & \int_{0}^{\pi} f(\theta) d \theta\left[4 F^{\prime \prime}(w)\left\{(a x+b y)^{2}+(h x+b y)^{2}\right\}\right. \\
& \left.+2 F^{\prime}(w)\{a+b+u(a+h y / x)+v(b+h x / y)\}\right]
\end{aligned}
$$

where $w=a x^{2}+2 h x y+b y^{2}$. As there are many different ways in which this might perhaps be done, it is not yet known whether all the useful types of integral have already been found.

If the limits were $-\pi$ and $\pi$, we could satisfy the equation by making the integrand the sum of an exact differential and an odd function 
of $\theta$. When the limits are 0 and $\pi$, there is the possibility that part of the integral may be zero on account of an invariance in form but with sign changed when $\theta$ is replaced by $\pi-\theta$. There are other possibilities suggested by the theory of functional differential equations, but at present we shall consider only the case in which the equation is satisfied because the integrand is equal to $d\left[G F^{\prime}(w)\right]$ where $G$ depends only on $x, y$ and $\theta$ in such a way that $G F^{\prime}(w)$ has the same finite value at both limits. In this case the analysis depends only on the ordinary theory of exact differentials, and it is not necessary to enter into extensions of this idea and extensions of the theory of the adjoint differential equation. Such extensions are occasionally useful but are not hard to develop.

Using primes to denote derivatives of $a, b, h, f, w$ and $G$ with respect to $\theta$, the equations to be satisfied are

$$
\begin{aligned}
G w^{\prime} & =4 f\left(A^{2}+B^{2}\right), \quad A=a x+h y, B=h x+b y, \\
x y G^{\prime} & =2 f[(a+b) x y+u y A+v x B] .
\end{aligned}
$$

Eliminating $G$ we obtain an equation which can be satisfied only if $u h b^{\prime}=0$ and $v h a^{\prime}=0$. The three possibilities are (1) $u=0, a^{\prime}=0$, (2) $v=0, b^{\prime}=0$, (3) $h=0$.

In the first case $a$ is constant and the equations become

$$
y G^{\prime}=2 f[(a+b) y+v B], \quad G y=4 f\left(A^{2}+B^{2}\right) / w_{0}
$$

where $w_{0}=2 h^{\prime} x+b^{\prime} y$. Eliminating $y G$ we obtain the equation

$$
\begin{aligned}
4 w_{0}\left(h^{\prime} y A+B B^{\prime}\right. & \left.+\frac{1}{2} f^{\prime} A^{2} / f+\frac{1}{2} f^{\prime} B^{2} / f\right) \\
& =2 w_{0}^{\prime}\left(A^{2}+B_{0}^{2}\right)+w^{2}[(a+b) y+v B],
\end{aligned}
$$

which should hold for all values of $x$ and $y$. Choosing the ratio of these quantities in such a way that $w_{0}=0$, we obtain the equation

$$
\left(h^{\prime \prime} b^{\prime}-b^{\prime \prime} h^{\prime}\right)\left[\left(a b^{\prime}-2 h h^{\prime}\right)^{2}+\left(h b^{\prime}-2 b h^{\prime}\right)^{2}\right]=0 \text {. }
$$

The first factor vanishes when $h^{\prime} / b^{\prime}$ is a constant. The second factor vanishes when $(a+i h) b^{\prime}=2(h+i b) h^{\prime}$ or

$$
b=2(a+i h)-a+c(a+i h)^{2},
$$

where $c$ is an arbitrary constant.

Equating the coefficients of $x^{3}$ in (5), we get

$$
h^{\prime \prime} / h^{\prime}-f^{\prime} / f=(2-v) h h^{\prime} /\left(a^{2}+h^{2}\right) .
$$

Hence $f d \theta=d h\left(a^{2}+h^{2}\right)^{(v-1) / 2}$, the constant of integration being put to unity. 
An appropriate value of the constant $c$ may be found by choosing the ratio of $x / y$ in (5) so that $A^{2}+B^{2}=0$ but $w_{0} \neq 0$. The value $c=-1 / a$ makes $w=A^{2} / a$ and the integral becomes the well known integral of Poisson ${ }^{1}$ if we take $h=-i \cos \theta$.

If we try

$$
z=\int_{-\pi}^{\pi} H(x-i y \cos \theta) f(\theta) d \theta
$$

the equation $r+t+v q / y=0$ will be satisfied if

$$
0=\left[H^{\prime} f \sin \theta\right]_{-\pi}^{\pi}-\int_{-\pi}^{\pi} H^{\prime}\left[f^{\prime} \sin \theta-(f v-1) \cos \theta\right] d \theta
$$

The integral may be made to vanish by choosing $f$ so that $-f^{\prime} \sin \theta$ $+f(v-1) \cos \theta$ is an odd function of $\theta$. In the case when this function is $\sin ^{2 n+1} \theta$, the equation for the determination of $f$ is

$$
f^{\prime}(\theta) \sin \theta-(v-1) f(\theta) \cos \theta=\sin ^{2 n+1} \theta
$$

and the appropriate solution is

$$
f(\theta)=\sin ^{v-1} \theta \int_{0}^{\theta} \sin ^{2 n-v+1} \theta d \theta .
$$

If, for example, $v=1$ and $n=0$, we get $f(\theta)=\theta$ and the integral is zero. The device does not yield a new solution.

Passing now to the case $h=0$, the equations (4) become

$\left(4^{\prime \prime}\right) G\left(a^{\prime} x^{2}+b^{\prime} y^{2}\right)=4\left(a^{2} x^{2}+b^{2} y^{2}\right) f, G^{\prime}=[2(1+u) a+2(1+v) b] f$.

Elimination of $G$ gives an equation

$$
\begin{aligned}
& 4\left(a a^{\prime} x^{2}+b b^{\prime} y^{2}\right)\left(a^{\prime} x^{2}+b^{\prime} y^{2}\right)-2\left(a^{2} x^{2}+b^{2} y^{2}\right)\left(a^{\prime \prime} x^{2}+b^{\prime \prime} y^{2}\right) \\
& +2\left(f^{\prime} / f\right)\left(a^{2} x^{2}+b^{2} y^{2}\right)\left(a^{\prime} x^{2}+b^{\prime} y^{2}\right) \\
& =[(1+u) a+(1+v) b]\left(a^{\prime} x^{2}+b^{\prime} y^{2}\right)^{2}
\end{aligned}
$$

which must be satisfied for all values of $x$ and $y$. Choosing the ratio of $x$ and $y$ so that $a^{\prime} x^{2}+b^{\prime} y^{2}=0$, we find that

$$
\left(a^{\prime \prime} b^{\prime}-a^{\prime} b^{\prime \prime}\right)\left(a^{2} b^{\prime}-b^{2} a^{\prime}\right)=0 \text {. }
$$

The first factor vanishes when $b^{\prime} / a^{\prime}$ is constant, the second factor when $b^{\prime} / b^{2}=a^{\prime} / a^{2}$ or $b^{-1}=a^{-1}+$ const. Taking the constant to be unity, we find that if $a=\tan ^{2} \frac{1}{2} \theta, b=\sin ^{2} \frac{1}{2} \theta$. Equating coefficients of $x^{4}$

${ }^{1}$ Journal de l'École Polytechnique, vol. 12 (1821), pp. 215-248. See also R. Hoppe, Journal für die reine und angewandte Mathematik, vol. 58 (1861), pp. 369-373. 
in (12), we find that

$$
4\left(a^{\prime} / a\right)-2\left(a^{\prime \prime} / a^{\prime}\right)+2 f^{\prime} / f=(1+u)\left(a^{\prime} / a\right)+(1+v)\left(b a^{\prime} / a^{2}\right) .
$$

Now $b a^{\prime} / a^{2}=\cot \frac{1}{2} \theta$ and consequently $f=\left(\tan \frac{1}{2} \theta\right)^{u}\left(\sin \frac{1}{2} \theta\right)^{v-1}$. It may be verified that if $w=x^{2} \tan ^{2} \frac{1}{2} \theta+y^{2} \sin ^{2} \frac{1}{2} \theta=W^{2}$ and $F$ is a suitable function, which may even be zero for $w>c^{2}$,

$$
z=\int_{0}^{\pi} F(w) \tan ^{u} \frac{1}{2} \theta \sin ^{v-1} \frac{1}{2} \theta d \theta
$$

is indeed a solution of $r+t+u p / x+v q / y=0$ when $R(u+v)>0$. A particular case of this result is that if $x>0, R(m)>-\frac{5}{4}, R(k+m)>-1$,

$$
\int_{0}^{\pi} W^{-k-m} J_{k+m}(W) \sin ^{2 m} \frac{1}{2} \theta \tan ^{2 k+1} \frac{1}{2} \theta d \theta=2 x^{-k} K_{k}(x) y^{-m} J_{m}(y) .
$$

This result may be verified by using Lommel's expansion

$$
W^{-k-m} J_{k+m}(W)=\sum_{n=0}^{\infty}\left(-\frac{1}{2} y^{2} S^{2}\right)^{n} / n !(x T)^{-k-m-n} J_{k+m+n}(x T),
$$

in which $S=\sin \frac{1}{2} \theta, T=\tan \frac{1}{2} \theta$. Now by a well known formula

$$
\begin{array}{r}
\int_{0}^{\pi} J_{k+m+n}(x T) S^{2 m+2 n} T^{k+1-m-n} d \theta=2\left(\frac{1}{2} x\right)^{m+n} K_{k}(x) / \Gamma(m+n+1), \\
-1<R(k+m+n)<R\left(2 m+2 n+\frac{3}{2}\right) .
\end{array}
$$

Hence, if $R(k+m)>-1, R(k-m)<\frac{3}{2}, R(m)>-\frac{5}{4}$, the series

$$
\begin{aligned}
\sum_{n=0}^{\infty}\left(-\frac{1}{2} y^{2}\right)^{n} / n ! \int_{0}^{\pi} & S^{2 m+2 n} T^{2 k+1}(x T)^{-k-m-n} J_{k+m+n}(x T) d \theta \\
& =2 x^{-k} K_{k}(x) \sum_{n=0}^{\infty}\left(-y^{2}\right)^{n} 2^{-m-2 n} / n ! \Gamma(m+n+1) \\
& =2 x^{-k} K_{k}(x) y^{-m} J_{m}(y) .
\end{aligned}
$$

The formula (16) is obtained by changing the order of integration and summation. ${ }^{2}$

If in the integral (15) $w$ is taken as the new independent variable, the integrand should satisfy the partial differential equation (2) for all

${ }^{2}$ This may be justified by forming estimates of the remainders in the two series. When $u=0, v=1$, the integral (15) is useful in potential theory and gives integral expressions for many symmetrical potentials. A list of these is being prepared. One such expression occurs in the paper of E. G. Gallop, The distribution of electricity on the circular disc and spherical bowl, Quarterly Journal of Mathematics, vol. 21 (1886), pp. 229-256. 
values of the parameter $w$. If $r=\left(2 w x^{2}+2 x^{2} y^{2}-2 y^{2} w+x^{4}+y^{4}+w^{2}\right)^{1 / 2}$, the result is that

$$
z=r^{-1} x^{-2 k}\left(r+w-x^{2}-y^{2}\right)^{m+k}\left(r+w+x^{2}-y^{2}\right)^{-m}
$$

should be a solution of the partial differential equation. The solution of the partial differential equation (2) may be written in a more symmetrical form by putting

$$
w=\frac{x^{2}}{a^{2}+v}+\frac{y^{2}}{b^{2}+v}, \quad z=\int_{C} F(w)\left(a^{2}+v\right)^{-k-1}(b+v)^{-m-1} d v .
$$

The contour $C$ may start at infinity and enclose either $v=-a^{2}$ or $v=-b^{2}$. It may also run from $-\infty$ to $+\infty$ along a path which avoids the points $v=-a^{2}$ and $v=-b^{2}$. The solution is now seen to be related to the integral for the product of two Bessel functions obtained by S. O. Rice, Quarterly Journal of Mathematics (Oxford series), vol. 6 (1935), pp. 52-64.

This theorem may be extended to any number of variables of type $x$ and $y$. Thus if $w$ is defined by the equation

the integral

$$
w=\frac{x^{2}}{a^{2}+v}+\frac{y^{2}}{b^{2}+v}+\frac{z^{2}}{c^{2}+v},
$$

$$
V=\int F(w)\left(a^{2}+v\right)^{-\alpha-1}\left(b^{2}+v\right)^{-\beta-1}\left(c^{2}+v\right)^{-\gamma-1} d v
$$

may be a solution of the partial differential equation

$$
\begin{aligned}
0=V_{x x} & +V_{y y}+V_{z z}+(2 \alpha+1) x^{-1} V_{x} \\
& +(2 \beta+1) y^{-1} V_{y}+(2 \gamma+1) z^{-1} V_{z}
\end{aligned}
$$

if the contour starts from $\infty$ and surrounds one of the points $-a^{2}$, $-b^{2},-c^{2}$ and returns to $\infty$. The restrictions are that $\alpha, \beta$, and $\gamma$ must be such that the integral is convergent and the arbitrary func$F$ must be such that differentiations under the integral sign are permissible.

California Institute of Technology 